\title{
Analytical brightness compensation algorithm for traditional polygon-based method in computer-generated holography
}

\author{
Yijie Pan, ${ }^{1,2}$ Yongtian Wang, ${ }^{1,3}$ Juan Liu, ${ }^{1, *}$ Xin Li, ${ }^{1}$ Jia Jia, ${ }^{1}$ and Zhao Zhang ${ }^{1}$ \\ ${ }^{1}$ School of Optoelectronics, Beijing Institute of Technology, Beijing 100081, China \\ ${ }^{2}$ Bradley Department of Electrical and Computer Engineering, Virginia Tech, Blacksburg, Virginia 24061, USA \\ ${ }^{3}$ e-mail: wyt @ bit.edu.cn \\ ${ }^{*}$ Corresponding author: juanliu@ bit.edu.cn \\ Received 7 March 2013; accepted 12 April 2013; \\ posted 21 May 2013 (Doc. ID 186570); published 19 June 2013
}

\begin{abstract}
In three-dimensional (3D) holographic display, current brightness compensation algorithm of the traditional polygon-based method experimentally obtains the compensation factor, which depends on the fabrication process. In this paper, we proposed an analytical brightness compensation method discarding the influence of the fabrication. The surface property function with the flat power spectral density and the compensation factor obtained from the simplified relationship between the original and the rotated frequencies are used to analytically compensate the radiant energy of the tilted polygon. The optical reconstruction results show the proposed method could effectively compensate the brightness and ensure the further shading process. The proposed method separates the brightness compensation from the fabrication process, which is important for deepening the investigation of the hologram fabrication and achieving realistic 3D reconstruction. (C) 2013 Optical Society of America

OCIS codes: $\quad$ (090.0090) Holography; (090.2870) Holographic display; (090.1760) Computer holography; (000.3860) Mathematical methods in physics.

http://dx.doi.org/10.1364/AO.52.004391
\end{abstract}

\section{Introduction}

In recent years, there has been considerable interest in developing three-dimensional (3D) display. Among various approaches, holographic display is supposed to be a promising method since it could provide all the depth cues of a 3D scene [1]. Computer-generated holography $(\mathrm{CGH})$ is the digital method in the hologram synthesis. According to the sampling method, there are two main approaches in the CGH computation. One is the point-based method. The other is the polygon-based method. The point method samples the 3D object by enormous self-illuminated points. Although many researchers have developed

$1559-128 \mathrm{X} / 13 / 184391-09 \$ 15.00 / 0$

(C) 2013 Optical Society of America various accelerating methods [2-4], to achieve the solid effect, the point-based method needs tremendous computation. The polygon-based method is expected to be the fast computation method since it represents the $3 \mathrm{D}$ objects by many polygons, by which the amount of the computation unit is significantly decreased [5-15]. In addition, combining with the rendering algorithms of the computer graphics, the polygon-based method could easily add texture and shade to the $3 \mathrm{D}$ scene.

Since the angular spectrum of the plane wave decomposition (PWD) theory is convenient for the numerical computation [16], the polygon-based methods under the PWD frame have been intensively researched for many years. In recent years, there have been four main approaches under the PWD frame: the traditional polygon-based method [5-10], 
the full analytical method [11,12], the spatial approximate method $[13,14]$, and the $3 \mathrm{D}$ affine transformation method [ $[\overline{15}]$. The traditional method depicts the tilted polygon in its local coordinates, computes the corresponding Fourier spectrum, calculates the new frequency from the spatial 3D rotational transformation and rotates the spectrum to the global coordinates by the interpolation approach. Although the traditional method conducts one fast Fourier transform (FFT) for each polygon, which adds the computation, it could add texture and smooth shade for each independent facet and reconstruct realistic $3 \mathrm{D}$ scene $[6,10]$. The full analytical polygon-based method defines the unique primitive polygon and computes its original spectrum. It implicitly uses the Fourier analysis of the two-dimensional affine transformation, the $3 \mathrm{D}$ rotational transformation of the traditional method, and the angular spectrum propagation to analytically compute the optical field at the hologram plane from the original spectrum. It discards the FFT for each facet but needs extra diffusion and texture adding computation that both are very time consuming [11,12]. The spatial approximate method uses the precomputed optical field, which is diffracted from the basic polygonal aperture to approximately calculate the diffraction field of the tilted polygon, which has rotational, shifting, and scaling relationships with the basic one $[13,14]$. All the computations of this method are in the spatial domain, therefore it saves the FFT. However, its reconstruction distance is constrained. Besides, it cannot add texture for each independent facet either. The 3D affine transformation method defines the primitive polygon and computes its spectrum in advance. It uses the pseudoinversion matrix to calculate the parameters of the 3D affine transformation from the vertex vectors of the primitive and the tilted polygon. Using these parameters, it directly connects the spectrum of the primitive and that of the tilted polygon without implicit variable substitution [15]. It saves the FFT but still needs other algorithm to add the texture. In brief, compared with the other three methods, which sacrifice some performances, such as the texturing capability and the reconstruction distance to obtain the fast computation, the traditional polygon-based method is suitable for achieving the realistic reconstruction in the holographic display.

In computer graphics, ambient light is a nondirectional, fixed-intensity, and fixed-color virtual light that affects all the objects equally [17]. The ambient illumination is used to provide the basic color and the frame of the 3D objects. Without correct ambient illumination, the 3D scene will have unexpected shade which will affect adding further diffusion and reflection information. To avoid this unexpected brightness difference, Matsushima proposed a brightness compensation method [6]. He concluded the unexpected shade is caused by the difference among the angles between the normal of each tilted polygon and the observation direction, and he proposed the compensation factor to control the divergence of the brightness and the corresponding angle factor to compensate the brightness. However, the compensation factor is experimentally determined instead of analytically derived, which depends on the actual methods for fabricating the hologram. In the case that the $3 \mathrm{D}$ scene has few polygons and there are large angles between each pair of them, it is hard to find out an appropriate compensation factor to eliminate the brightness differences. Further, with the intensive investigation of various fabrication processes, it is urgent to separate the CGH computation from the fabrication and independently research the influence of the later one.

In this paper, we regard the unexpected shade as a result of energy loss caused by the spectrum rotation, specifically the zero-padding approach of the interpolation method, in the traditional polygon-based method. We propose an analytical brightness compensation method for the traditional method. A surface property function that has the flat power spectral density (PSD) is used. For each titled polygon, the spatial rotational transformation between the global and its local coordinates is simplified to two independent rotations about the coordinate axes, by which the original and the new frequencies have finite and solvable relationship. The new frequency is approximated by discarding the higher-order infinitesimal term. The compensation factor is obtained from the geometric relationship between the original and the approximated new frequencies. When the spectrum is rotated, the radiant energy of the polygon is preserved by multiplying the compensation factor. The optical experiments show the proposed method could correctly compensate the brightness in the hologram synthesis. It could compute the compensation factor analytically instead of experimentally and separate the brightness compensation from the fabrication process.

The paper is organized as follows. In Section 2, the basic theories of the traditional polygon-based method and the proposed brightness compensation approach are presented. In Section 3, the optical experimental results are illustrated to show the correctness of the proposed method. In Section 4, some discussions about the proposed method are provided. In Section $\underline{5}$, we conclude the method with the future plan.

\section{Theory}

\section{A. Traditional Polygon-Based Method}

As illustrated in Fig. 1, for the rotation of each tilted polygon in the 3D scene, two coordinate systems are investigated. One is the local coordinate system $\left(x^{\prime}, y^{\prime}, z^{\prime}\right)$. The other is the global coordinate system $(x, y, z)$. They share the same origin $O$.

In the 3D Euclidian space, there always is a 3D rotational transformation between the two coordinates, which could transfer the tilted polygon $g(x, y, z)$ under the global coordinates to an original polygon $f\left(x^{\prime}, y^{\prime}, z^{\prime}=0\right)$ under the local coordinates by 


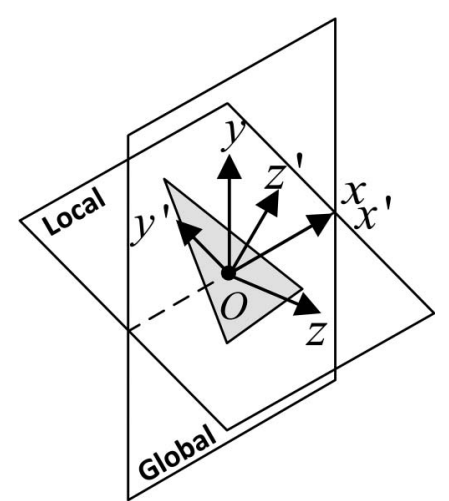

Fig. 1. Local and the global coordinates of a tilted polygon.

$$
\left(x^{\prime}, y^{\prime}, z^{\prime}\right)^{\prime}=T_{y x z} \times(x, y, z)^{\prime},
$$

where $\times$ represents the vector multiplication, $(\cdot)^{\prime}$ denotes the transverse operation. $T_{y x z}$ is an orthogonal $3 \mathrm{D}$ rotational transformation matrix, which is defined as [18]

$$
T_{y x z}=R_{y} \times R_{x} \times R_{z}=\left|\begin{array}{lll}
a_{1} & a_{2} & a_{3} \\
a_{4} & a_{5} & a_{6} \\
a_{7} & a_{8} & a_{9}
\end{array}\right|,
$$

where $R_{y}, R_{x}, R_{z}$ are the independent rotation matrices about the $y, x, z$ axes, respectively. where $\mu^{\prime}, \nu^{\prime}$ is the frequency along the $x^{\prime}, y^{\prime}$ axes, respectively.

Under the global coordinates, the angular spectrum of the tilted polygon at the $z=0$ plane is [16]

$$
G(\mu, \nu)=\iint g(x, y, z) e^{-i 2 \pi(\mu x+\nu y+\varpi z)} \mathrm{d} x \mathrm{~d} y,
$$

where $\varpi$ is the frequency about the $z$ axis, which is calculated by

$$
\varpi=\left(\lambda^{-2}-\mu^{2}-\nu^{2}\right)^{1 / 2} .
$$

From Eq. (1) and the orthogonal characteristic of $T_{y x z}$, the phase exponent $\mu x+\nu y+\varpi z$ in Eq. ( $\left.\underline{6}\right)$ can be written into

$$
\begin{aligned}
\mu x+\nu y+\varpi z= & \left(a_{1} \mu+a_{2} \nu+a_{3} \varpi\right) x^{\prime} \\
& +\left(a_{4} \mu+a_{5} \nu+a_{6} \varpi\right) y^{\prime} \\
& +\left(a_{7} \mu+a_{8} \nu+a_{9} \varpi\right) z^{\prime} .
\end{aligned}
$$

Substituting Eqs. (3) and (ㅁ) into Eq. (ㅁ) and changing the domain of integration from $x, \bar{y}$ to $x^{\prime}$, $y^{\prime}$, the spectrum of the tilted polygon is

$$
\begin{aligned}
G(u, v) & =\iint g(x, y, z) e^{-j 2 \pi(\mu x+\iota y+\varpi z)} \mathrm{d} x \mathrm{~d} y \\
& =J \cdot \iint f\left(x^{\prime}, y^{\prime}, z^{\prime}=0\right) e^{-j 2 \pi\left[\left(a_{1} \mu+a_{2} \nu+a_{3} \varpi\right) x^{\prime}+\left(a_{4} \mu+a_{5} \nu+a_{6} \varpi\right) y^{\prime}+\left(a_{7} \mu+a_{8} \nu+a_{9} \varpi\right) z^{\prime}\right]} \mathrm{d} x^{\prime} \mathrm{d} y^{\prime} \\
& =J \cdot \iint f\left(x^{\prime}, y^{\prime}, z^{\prime}=0\right) e^{-j 2 \pi\left(\mu^{\prime \prime} x^{\prime}+\nu^{\prime \prime} y^{\prime}+\varpi^{\prime \prime} z^{\prime}\right)} \mathrm{d} x^{\prime} \mathrm{d} y^{\prime}=J \cdot F\left(\mu^{\prime \prime}, \nu^{\prime \prime}\right),
\end{aligned}
$$

According to Eq. (1), the expressions of the tilted and the original polygon have the relationship

$$
\begin{aligned}
g(x, y, z)= & f\left(a_{1} x+a_{2} y+a_{3} z, a_{4} x+a_{5} y+a_{6} z, a_{7} x\right. \\
& \left.+a_{8} y+a_{9} z\right)=f\left(x^{\prime}, y^{\prime}, z^{\prime}=0\right) .
\end{aligned}
$$

Under the local coordinates, the original polygon is represented by its surface function

$$
f\left(x^{\prime}, y^{\prime}, z^{\prime}=0\right)=a\left(x^{\prime}, y^{\prime}\right) \psi\left(x^{\prime}, y^{\prime}\right)
$$

where $a\left(x^{\prime}, y^{\prime}\right)$ is the amplitude function, which is a real function representing the texture and shade information. $\psi\left(x^{\prime}, y^{\prime}\right)$ is the phase function, which carries diffusive and reflective information. The surface function and its Fourier transform have the relationship

$$
F\left(\mu^{\prime}, \nu^{\prime}\right)=\iint f\left(x^{\prime}, y^{\prime}, z^{\prime}=0\right) e^{-j 2 \pi\left(\mu^{\prime} x^{\prime}+\nu^{\prime} y^{\prime}\right)} \mathrm{d} x^{\prime} \mathrm{d} y^{\prime},
$$

where $\cdot$ represents the scalar multiplication, $\mu^{\prime \prime}, \nu^{\prime \prime}$, $\varpi^{\prime \prime}$ is the new frequency, and $J$ is the Jacobian of the change of the integration domain.

In Eq. (9), the new and the original frequencies satisfy

$$
\left(\mu^{\prime \prime}, \nu^{\prime \prime}, \varpi^{\prime \prime}\right)^{\prime}=T_{y x z} \times(\mu, \nu, \varpi)^{\prime} .
$$

The Jacobian of the change of the integration domain can be obtained from Eq. (1):

$$
J=\left|\begin{array}{ll}
\frac{\partial x^{\prime}}{\partial x} & \frac{\partial x^{\prime}}{\partial y} \\
\frac{\partial y^{\prime}}{\partial x} & \frac{\partial y^{\prime}}{\partial y}
\end{array}\right|=\left|a_{1} a_{5}-a_{2} a_{4}\right| .
$$

In order to make the spectral domain of the local and the global coordinates share the same origin point, the traditional method introduces the frequency offset

$$
\mu_{0}=a_{3} / \lambda \quad \nu_{0}=a_{6} / \lambda .
$$


Substituting Eq. (2) into Eq. (10) and subtracting the offset from the new frequency, we obtain

$$
\begin{aligned}
\mu^{\prime \prime \prime} & =a_{1} \mu+a_{2} \nu+a_{3} \varpi(\mu, \nu)-\mu_{0} \\
\nu^{\prime \prime \prime} & =a_{4} \mu+a_{5} \nu+a_{6} \varpi(\mu, \nu)-\nu_{0} .
\end{aligned}
$$

The spectrum of the arbitrary polygon under the global local coordination will be

$$
G(u, v) \approx J \cdot F\left(\mu^{\prime \prime \prime}, \nu^{\prime \prime \prime}\right),
$$

where the sign for nearly equal means the interpolation is used.

In brief, we can calculate the global spectrum of the tilted polygon from the spectrum of the original polygon under the local coordinates and the 3D transformation matrix between the two coordinates. After propagation and superimposition of the spectrum of each titled polygon under the global coordinates, we can get the hologram in the spatial domain by the inverse Fourier transform.

\section{B. Brightness Compensation}

In the radiometry, the radiance of a surface is defined as

$$
L=\frac{d^{2} \Phi}{d A d \Omega \cos \theta_{v}}=\frac{d I}{d A \cos \theta_{v}},
$$

where $d(\cdot)$ represents an infinitely small change in the variables. $\Phi$ represents the radiant flux of the surface. $A$ denotes the area of the surface. $\Omega$ is the solid angle of the emission of the surface. $\theta_{v}$ is angle between the observation direction and the surface normal. $I$ represents the radiant intensity of the surface.

The radiance of an ideal diffusive reflecting surface, the Lambert surface, is constant regardless of the observation direction since the radiant intensity is proportional to the cosine of the angle between the view direction and the surface normal. In accordance to Eq. (15) and the isotopic characteristic, for the fixed area and the fixed solid angle, the radiance of the Lambert surface is directly proportional to the radiant flux of the surface.

Let the $h(x, y, z)$ represent the radiant function of the surface and its Fourier transform be $H(\mu, \nu)$. The PSD is a numerical characteristic of a stochastic signal, which is the power distribution over frequency. For $h(x, y, z)$ signal, it is $|H(\mu, \nu)|^{2}$. In accordance to the Parseval theorem, the radiant flux of the surface can be calculated by integrating its PSD in the frequency domain as

$$
\begin{aligned}
\Phi & =\iint_{D 1}|h(x, y)|^{2} \mathrm{~d} x \mathrm{~d} y=\iint_{D 2}|H(\mu, \nu)|^{2} \mathrm{~d} \mu \mathrm{d} \nu \\
& =\iint_{D 2} P S D(\mu, \nu) \mathrm{d} \mu \mathrm{d} \nu,
\end{aligned}
$$

where $P S D(\mu, \nu)$ denotes the PSD of the surface function, $D 1$ is the domain of the spatial function, $D 2$ is the domain of the spectrum.
We choose the white noise signal, which has the flat PSD as the phase function. That means the signal contains equal power within a fixed bandwidth at any center frequency. The surface signal $f\left(x^{\prime}, y^{\prime}, z^{\prime}=\right.$ 0 ) is also a white noise signal since it is the function of $\psi\left(x^{\prime}, y^{\prime}\right)$. Let the PSD of the surface function equal $P$ which is a constant real number. Substituting the Fourier pair of the surface function and the constant PSD into Eq. (16), the radiant flux of the surface is

$$
\Phi_{\text {orig }}=P \cdot S_{\text {orig }},
$$

where $S_{\text {orig }}$ is the area of the domain of the original spectrum.

From Section 2.A, the essence of the traditional polygon-based method is calculating the analytic relationship between the new frequency $\left(\mu^{\prime \prime \prime}, \nu^{\prime \prime \prime}\right)$ and the original frequency $(\mu, \nu)$ by the use of the 3D rotational transformation and remapping the original spectrum to the new frequency using the interpolation approach. In the numerical computation, when the new frequency exceeds the original domain, the interpolation method uses zero-padding approach to fill the new spectrum, which means only the spectrum on the public of the new and the original frequency is actually used in the interpolation. In this case, the spectrum loss equals the energy loss, which exhibits the brightness difference in the reconstruction.

We name the public region of the new and the original domain as the effective domain. In Fig. $\underline{2}$, we illustrate the relationships among those domains without loss of generality. The square ABCD is the original frequency domain. The curved edge shape $\mathrm{A}^{\prime} \mathrm{B}^{\prime} \mathrm{C}^{\prime} \mathrm{D}^{\prime}$ is the new frequency domain, which intersects the original domain at points $E^{\prime}, F^{\prime}, G^{\prime}, H^{\prime}$. Therefore, the effective domain is the shape $\mathrm{E}^{\prime} \mathrm{A}^{\prime} \mathrm{F}^{\prime} \mathrm{G}^{\prime} \mathrm{C}^{\prime} \mathrm{H}^{\prime}$. The dashed-lined parallelogram $\mathrm{A}^{\prime \prime} \mathrm{B}^{\prime \prime} \mathrm{C}^{\prime \prime} \mathrm{D}^{\prime \prime}$, which intersects the original domain at $E^{\prime \prime}, F^{\prime \prime}, G^{\prime \prime}, H^{\prime \prime}$, will be discussed in the following.

Since the interpolation does not change the PSD and it uses zero-padding when the new frequency

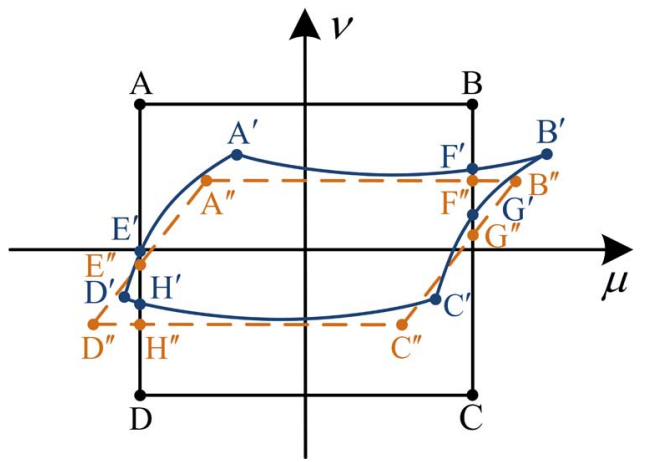

Fig. 2. Original, the new and the approximated new frequency domains. The square $A B C D$ represents the original domain. The curved edge shape $\mathrm{A}^{\prime} \mathrm{B}^{\prime} \mathrm{C}^{\prime} \mathrm{D}^{\prime}$ defines the new frequency domain. The shape $\mathrm{E}^{\prime} \mathrm{A}^{\prime} \mathrm{F}^{\prime} \mathrm{G}^{\prime} \mathrm{C}^{\prime} \mathrm{H}^{\prime}$ is the effective domain. The parallelogram $\mathrm{A}^{\prime \prime} \mathrm{B}^{\prime \prime} \mathrm{C}^{\prime \prime} \mathrm{D}^{\prime \prime}$ represents the approximated new frequency domain. The shape $\mathrm{E}^{\prime \prime} \mathrm{A}^{\prime \prime} \mathrm{F}^{\prime \prime} \mathrm{G}^{\prime \prime} \mathrm{C}^{\prime \prime} \mathrm{H}^{\prime \prime}$ is the approximated effective domain. 
domain exceeds the original one, after the spectrum rotation, the radiant flux of the new signal is

$$
\Phi_{\text {new }}=P \cdot S_{\text {eff }},
$$

where $S_{\text {eff }}$ is the area of the effective domain.

We define a real positive energy compensation factor $C$. The compensated spectrum is

$$
G(\mu, \nu)^{\prime}=G(\mu, \nu) / C .
$$

Substituting Eq. (19) into Eq. (16), the radiant flux of the compensated new spectrum is

$$
\Phi_{\text {comp }}=\iint_{D 2}|G(\mu, \nu) / C|^{2} \mathrm{~d} \mu \mathrm{d} \nu=P \cdot S_{\text {eff }} / C^{2} .
$$

Combining Eqs. (20) and (17) and letting the compensated radiant flux equal to the original one, we get

$$
C=\left(S_{\text {eff }} / S_{\text {orig }}\right)^{1 / 2}
$$

From above, we conclude that we can compensate the brightness of the rotated polygon by calculating the square root of the ratio between the area of the effective and the original domain. Next we will compute the area of the effective domain.

In Eq. (2), the multiplication of the three matrices $R_{y} \times R_{x} \times \bar{R}_{z}$ expresses the sequence of the rotation about the axes. That is, the first rotation is about the $z$ axis, and the last rotation is about the $x$ axis. Since the first $z$ rotation does not change the surface normal of the original polygon, we could always temporally ignore it and find out a pair of rotation angle $\alpha, \beta$ about the $x, y$ axes within $\left[-90^{\circ}, 90^{\circ}\right]$ in accordance to the relationship between the surface normal of the original polygon and that of the tilted polygon. The ignorance of the $z$ rotation is by letting $R_{z}$ equal the identity matrix. Then the rotation matrix is simplified to $T_{y x}=R_{y} \times R_{x}$. The independent rotation matrices $R_{x}$ and $R_{y}$ are

$$
\begin{aligned}
& R_{x}=\left[\begin{array}{ccc}
1 & 0 & 0 \\
0 & \cos \alpha & -\sin \alpha \\
0 & \sin \alpha & \cos \alpha
\end{array}\right], \\
& R_{y}=\left[\begin{array}{ccc}
\cos \beta & 0 & \sin \beta \\
0 & 1 & 0 \\
-\sin \beta & 0 & \cos \beta
\end{array}\right] .
\end{aligned}
$$

Plugging Eq. (22) into Eq. (2), $T_{y x}$ could be

$$
T_{y x}=\left[\begin{array}{ccc}
\cos \beta & \sin \alpha \sin \beta & \cos \alpha \sin \beta \\
0 & \cos \alpha & -\sin \alpha \\
-\sin \beta & \sin \alpha \cos \beta & \cos \alpha \cos \beta
\end{array}\right] .
$$

Substituting Eq. (23) into Eq. (13), we obtain

$$
\begin{aligned}
& \mu^{\prime \prime \prime}=\mu \cos \beta+\nu \sin \alpha \sin \beta+a_{3} \varpi-\mu_{0} \\
& \nu^{\prime \prime \prime}=\nu \cos \alpha+a_{6} \varpi-\nu_{0} .
\end{aligned}
$$

Expanding $\varpi$ from Eq. () and substituting Eq. (12) into Eq. (24), we obtain

$$
\begin{aligned}
\mu^{\prime \prime \prime} & =\mu \cos \beta+\nu \sin \alpha \sin \beta+o(\mu, \nu) \\
\nu^{\prime \prime \prime} & =\nu \cos \alpha+o(\mu, \nu),
\end{aligned}
$$

where $o(\mu, \nu)$ represents the higher-order infinitesimal terms of $\mu, \nu$. It is an even function of $\mu, \nu$. Under paraxial condition, compared with $\mu, \nu, o(\mu, \nu)$ is very close to zero. Then we discard $o(\mu, \nu)$ in Eq. (25) and define the approximated new frequency by

$$
\begin{aligned}
& \hat{\mu}=\mu \cos \beta+\nu \sin \alpha \sin \beta \\
& \hat{\nu}=\nu \cos \alpha .
\end{aligned}
$$

In Fig. 2, the dashed-lined parallelogram $\mathrm{A}^{\prime \prime} \mathrm{B}^{\prime \prime} \mathrm{C}^{\prime \prime} \mathrm{D}^{\prime \prime}$ represents the approximated new frequency domain, which intersects the original domain at $\mathrm{E}^{\prime \prime}, \mathrm{A}^{\prime \prime}, \mathrm{F}^{\prime \prime}, \mathrm{G}^{\prime \prime}, \mathrm{C}^{\prime \prime}, \mathrm{H}^{\prime \prime}$. According to the characteristic of $o(\mu, \nu)$, the area of the shape $\mathrm{A}^{\prime \prime} \mathrm{B}^{\prime \prime} \mathrm{C}^{\prime \prime} \mathrm{D}^{\prime \prime}$ equates to the area of the shape $\mathrm{A}^{\prime} \mathrm{B}^{\prime} \mathrm{C}^{\prime} \mathrm{D}^{\prime}$, and the areas of $\mathrm{E}^{\prime} \mathrm{A}^{\prime} \mathrm{F}^{\prime} \mathrm{G}^{\prime} \mathrm{C}^{\prime} \mathrm{H}^{\prime}$ equals the area of $\mathrm{E}^{\prime \prime} \mathrm{A}^{\prime \prime} \mathrm{F}^{\prime \prime} \mathrm{G}^{\prime \prime} \mathrm{C}^{\prime \prime} \mathrm{H}^{\prime \prime}$. We name the public between the approximated new and the original frequency domain, shape $\mathrm{E}^{\prime \prime} \mathrm{A}^{\prime \prime} \mathrm{F}^{\prime \prime} \mathrm{G}^{\prime \prime} \mathrm{C}^{\prime \prime} \mathrm{H}^{\prime \prime}$, as the approximated effective domain.

According to Eq. (26), the absolute value of $\hat{\nu}$ is not greater than that of $\nu$. Therefore, the relationship between the approximated new and the original frequency domain is determined by the horizontal relationship between the $\mu$ coordinates of the vertices of the two shapes. We only investigate points $\mathrm{A}^{\prime}, \mathrm{A}^{\prime \prime}$ and $\mathrm{B}^{\prime}, \mathrm{B}^{\prime \prime}$ since both two domains are symmetrical about the origin point. Let the coordinates of the points $\mathrm{A}^{\prime}$ and $\mathrm{B}^{\prime}$ be $(-m, m)$ and $(m, m)$, respectively. Since both $\alpha$ and $\beta$ are under the scope of $\left[-90^{\circ}, 90^{\circ}\right]$, it can be proved that $\hat{\mu}_{\mathrm{A}^{\prime \prime}} \in[-\sqrt{2} m, m], \hat{\mu}_{\mathrm{B}^{\prime \prime}} \in[-m, \sqrt{2} m]$ and the absolute value of them, $\left|\hat{\mu}_{\mathrm{A}^{\prime \prime}}\right|$ and $\left|\hat{\mu}_{\mathrm{B}^{\prime \prime}}\right|$, could not be greater than $m$ at the same time. Then, we conclude that there are three relationships between the two domains, which are featured in: (a) $\left|\hat{\mu}_{\mathrm{B}^{\prime \prime}}\right|>m$ and $\left|\hat{\mu}_{\mathrm{A}^{\prime \prime}}\right|<m$, (b) $\left|\hat{\mu}_{\mathrm{B}^{\prime \prime}}\right|<m$ and $\left|\hat{\mu}_{\mathrm{A}^{\prime \prime}}\right|>m$, (c) $\left|\hat{\mu}_{\mathrm{B}^{\prime \prime}}\right|<m$ and $\left|\hat{\mu}_{\mathrm{A}^{\prime \prime}}\right|<m$. Figure 3 demonstrates the corresponding graphic relationships. In Fig. 3, for conditions (a), (b), and (c), $S_{\text {eff }}$ is the area of shape $\mathrm{A}^{\prime \prime} \mathrm{F}^{\prime \prime} \mathrm{G}^{\prime \prime} \mathrm{C}^{\prime \prime} \mathrm{H}^{\prime \prime} \mathrm{E}^{\prime \prime}, \mathrm{F}^{\prime \prime} \mathrm{B}^{\prime \prime} \mathrm{G}^{\prime \prime} \mathrm{H}^{\prime \prime} \mathrm{D}^{\prime \prime} \mathrm{E}^{\prime \prime}$, and $\mathrm{A}^{\prime \prime} \mathrm{B}^{\prime \prime} \mathrm{C}^{\prime \prime} \mathrm{D}^{\prime \prime}$, respectively. $S_{\text {orig }}$ is the area of square ABCD. Finally, for the three conditions, we can obtain the compensation factors in accordance with Eq. (21): 


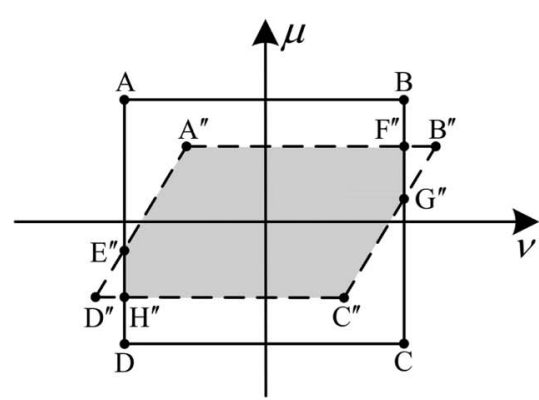

(a)

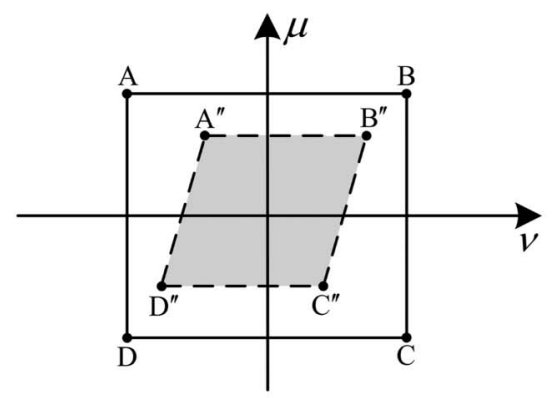

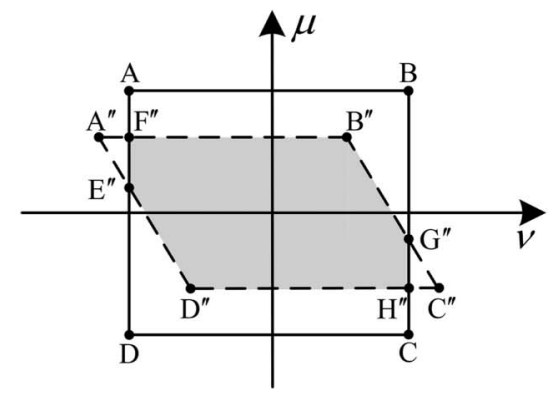

(b)

(c)

Fig. 3. Three geometric relationships between the approximated new and the original frequency domain. The gray area is the corresponding approximated effective domain. (a) One pair of the vertices of the parallelogram B" $\mathrm{D}^{\prime \prime}$ is outside the square. (b) The other pair of the vertices of the parallelogram $\mathrm{A}^{\prime \prime} \mathrm{C}^{\prime \prime}$ is outside the square. (c) All the vertices of the parallelogram are inside the square.

$$
C=\left\{\begin{array}{cl}
\left.\cos \alpha-\mid \frac{(1+\sin \alpha \sin \beta-\cos \beta)^{2}}{4 \sin \alpha \sin \beta}\right) \mid \cos \alpha & \text { for relation }(a) \\
\left.\cos \alpha-\mid \frac{(\cos \beta+\sin \alpha \sin \beta-1)^{2}}{4 \sin \alpha \sin \beta}\right) \mid \cos \alpha & \text { for relation }(b) \\
\cos \alpha \cos \beta & \text { for relation }(c)
\end{array}\right.
$$

\section{Experimental Results}

In this section we will present some visual results from our proof-of-concept implementation. As illustrated in Fig. 4, the $671 \mathrm{~nm}$ diode pump solid-state laser, the spatial filter, the collimating lens, and the half-wave plate are used. The reflective phase-only spatial light modulator (SLM: Holoeye Pluto-VIS, resolution $1920 \times 1080$, pixel size $8 \mu \mathrm{m}$ ) is used to modulate the oblique incident plane wave. It is located at the front focus of a Fourier lens $(\mathrm{FL} ; \Phi=120 \mathrm{~mm} f=543 \mathrm{~mm})$. A high-pass filter (HPF) is placed at the rear focus of the FL to suppress the zero order caused by the pixelated SLM.

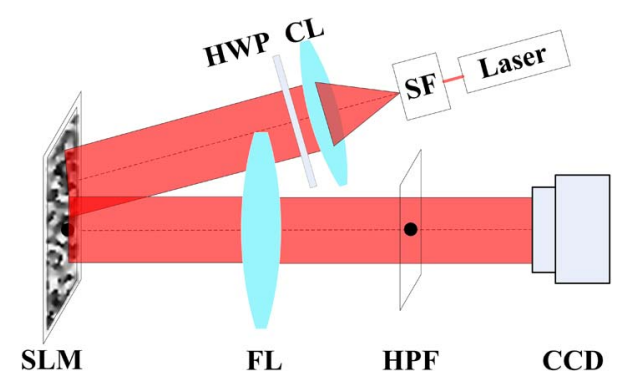

Fig. 4. Experimental setup: SF, spatial filter; CL, collimating lens; HWP, half-wave plate; SLM, spatial light modulator; FL, Fourier lens; HPF, high-pass filter; CCD, CCD camera.
A 3D scene is reconstructed behind the HPF, which is captured by a CCD camera (Lumenera INFINITY 4-11C pixel size $9 \mu \mathrm{m}$ ). A Fourier transform hologram is loaded on the SLM and the FL imposes an inverse Fourier transform to the modulated wavefront.

We reconstruct two sets of $3 \mathrm{D}$ scenes. As illustrated in Figs. 5(a)-5(d), a cubic and a pyramid are reconstructed. In Figs. 5(e)-5(h), a cylinder whose lateral is equally divided into eighteen segments is reconstructed. In Figs. 5(a) and 5(e), no brightness compensation algorithm is used. The reconstructed 3D scenes have unexpected brightness difference. The public edges between the polygons pairs have brightness differences that are observable. The two scenes are reconstructed as if the ob-

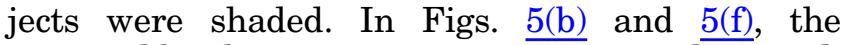
proposed brightness compensation approach is used. The reconstructed scenes have only boundary and color information as we expected. In Figs. 5(c) and $5(\mathrm{~g})$, the proposed compensation method and the flat shading method are used. One parallel light with the direction vector equaling $(-\sqrt{2},-1,-1)$ is used to illuminate the two 3D scenes, respectively. In Figs. 5(d) and 5(h), the same approaches are explored except the direction vector of the parallel light is $(\sqrt{2},-1,-1)$. As shown in Figs. 5(c), 5(d), 5(g), and 5 (h), the reconstructed images have correct shade information.

\section{Discussion}

From the experimental results in Fig. 5, it is obvious that the proposed method could effectively 

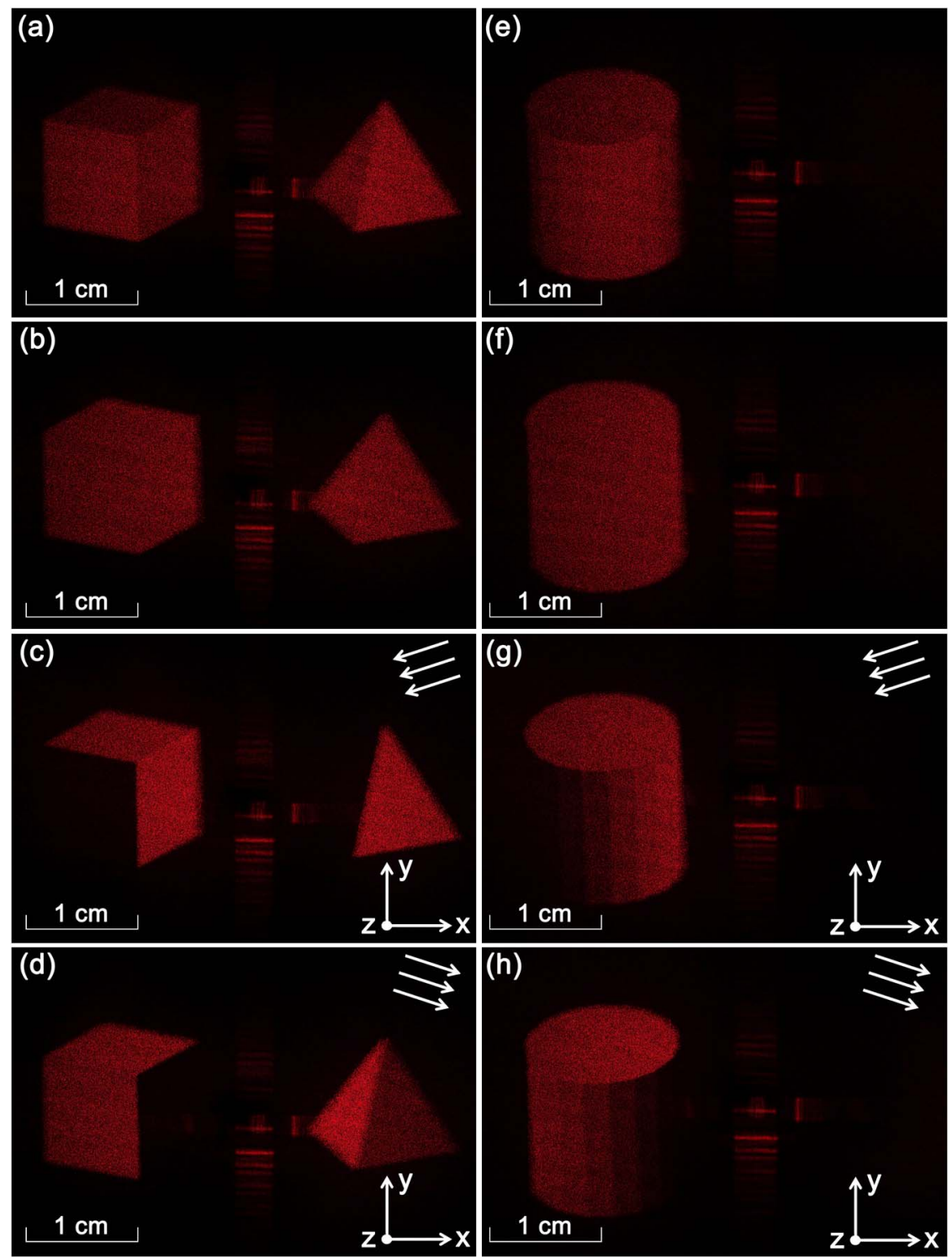

Fig. 5. Two sets of optical reconstruction results. (a) and (e) are results without the compensation method. (b) and (f) are results with the proposed compensation approach. (c), (g) and (d), (h) are two pairs of reconstruction results with the proposed brightness compensation method and the illumination by the parallel lights with light vectors equaling $(-\sqrt{2},-1,-1)$ and $(\sqrt{2},-1,-1)$, respectively.

compensate the unexpected brightness difference caused by the spectrum rotation, and it ensures the further shading process.

It is important to note, the proposed method will not affect the texture mapping process of the traditional polygon-based method. In the texture mapping process, the texture polygon is under the local coordinates and is given in accordance to the rotation matrix $T_{y x z}$. In Eq. (2), the rotation about the $z$ axis could be represent by the matrix

$$
R_{z}=\left[\begin{array}{ccc}
\cos \gamma & -\sin \gamma & 0 \\
\sin \gamma & \cos \gamma & 0 \\
0 & 0 & 1
\end{array}\right],
$$

where $\gamma$ denotes the rotation angle.
Supposing one vertex of the tilted polygon has a vertex vector $v p a$ and the corresponding vertex vector of the texture polygon is $v p a^{\prime \prime}$, they satisfy

$$
v p a^{\prime \prime}=T_{y x} \times R_{z} \times v p a .
$$

Therefore, we could obtain the rotation angle $\gamma$ from $v p a, v p a^{\prime \prime}, \alpha$ and $\beta$, by which we can correctly map the texture to the tilted polygon.

We present an experimental result of the texture mapping to a $3 \mathrm{D}$ object with the proposed method. The 3D model consists of a cubic that rotates $45^{\circ}$ about both the $x$ and $y$ axes. The three surfaces confronting the observer are textured with three letters. The actual texture polygons are six isosceles triangles. We computed the hologram with the proposed method but no directional illumination. In Fig. $\underline{6}$, 


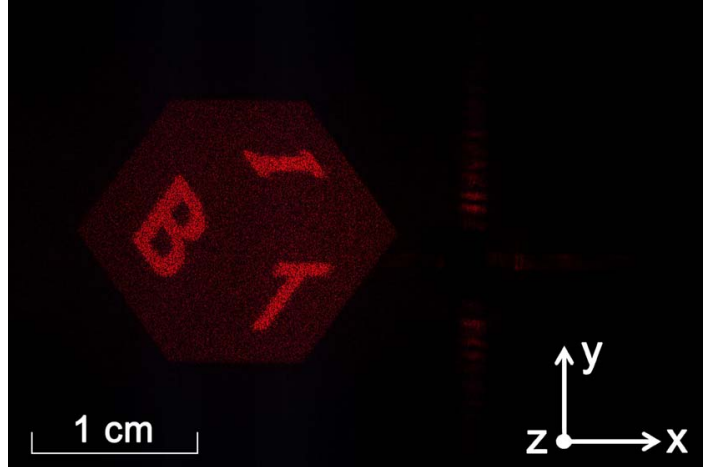

Fig. 6. Optical reconstruction of the traditional polygon-based method with the proposed brightness compensation method and the texture approach.

the reconstruction result shows the brightness is correctly compensated and the textures are effectively added.

Next we will discuss whether we could discard the higher-order term in Eq. (25). We conclude that it depends on the reconstruction wavelength and the spectral bandwidth of the hologram.

We expand Eq. (7) as

$$
\begin{aligned}
\varpi= & \frac{1}{\lambda}\left(1-\lambda^{2} \mu^{2}-\lambda^{2} \nu^{2}\right) \\
= & \frac{1}{\lambda}+\left[-\frac{\lambda u^{2}+\lambda \nu^{2}}{2}\right]+\left[-\frac{\left(\lambda u^{2}+\lambda \nu^{2}\right)^{2}}{8}\right]+\ldots \\
& +\frac{1}{(n+1) !} f^{n+1}(0)\left(\lambda u^{2}+\lambda \nu^{2}\right)^{n+1} .
\end{aligned}
$$

Since $\left[-\left(\lambda u^{2}+\lambda \nu^{2} / 2\right)\right]$ contains much value of the remainder, the following proof will focus on this term.

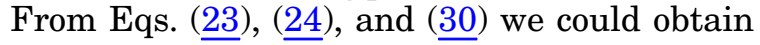

$\mu^{\prime \prime \prime}=\mu \cos \beta+\nu \sin \alpha \sin \beta-\cos \alpha \sin \beta \frac{\lambda u^{2}+\lambda \nu^{2}}{2}$

$\nu^{\prime \prime \prime}=\nu \cos \alpha+\sin \alpha \frac{\lambda u^{2}+\lambda \nu^{2}}{2}$.

The higher-order term brings distortion to the parallelogram $\mathrm{A}^{\prime \prime} \mathrm{B}^{\prime \prime} \mathrm{C}^{\prime \prime} \mathrm{D}^{\prime \prime}$. We investigate whether the $\nu^{\prime \prime \prime}$ values of the vertices of the new frequency domain exceed the range of the $\nu$ values of the original domain and whether the excessive amount could be neglected.

For square original domain, from Eq. (31), we obtain

$$
\begin{aligned}
\frac{\nu_{\mathrm{B}^{\prime \prime}}^{\prime \prime \prime}-\nu_{\mathrm{B}}}{\nu_{\mathrm{B}}} & =\cos \alpha+\lambda \nu_{\mathrm{B}} \sin \alpha-1 \\
& =\left(1+\lambda^{2} \nu_{\mathrm{B}}^{2}\right)^{1 / 2} \sin (\psi+\alpha)-1,
\end{aligned}
$$

where angle $\psi$ satisfies $\sin \psi=\left(1+\lambda^{2} \nu_{\mathrm{B}}^{2}\right)^{-1 / 2}$ and $\cos \psi=\lambda \mu\left(1+\lambda^{2} \nu_{\mathrm{B}}^{2}\right)^{-1 / 2}$. It is clear, the maximum value of Eq. $(\underline{32})$ is $\left(1+\lambda^{2} \nu_{\mathrm{B}}^{2}\right)^{1 / 2}-1$.
We first test the extreme condition where $\lambda^{2} \nu^{2}$ equals 0.5 , the maximum value of $\left(\nu_{\mathrm{B}^{\prime \prime}}^{\prime \prime \prime}-\nu_{\mathrm{B}}\right) / \nu_{\mathrm{B}}$ is 0.225 . That means the $\nu^{\prime \prime \prime}$ values of the vertices will exceed the original $\nu$ value by $22.5 \%$. In this case, we cannot discard the higher-order term in Eq. (25) and we cannot compute the area of the effective domain since the curved new frequency domain has severe distortion.

In the previous optical experiments, the wavelength of the laser beam is $671 \mathrm{~nm}$ and the pitch of the SLM is $8 \mu \mathrm{m}$. That is, $\lambda \nu$ equals 0.0419 . From Eq. (32), we can compute the maximum $\left(\nu_{\mathrm{B}^{\prime \prime}}^{\prime \prime \prime}-\nu_{\mathrm{B}}\right) / \nu_{\mathrm{B}}$ is only 0.0008 . From Figs. 5 and 6 , it is clear that discarding the higher-order term will not influence the reconstruction image quality. That is because the excessive amount is too small. Likewise, we can prove this conclusion also works on the $\mu^{\prime \prime \prime}$ values of the new frequency domain.

From above proof, eliminating the higher-order term depends on the product of the laser wavelength and the spectral bandwidth of the hologram. Excessive long wavelength or wide bandwidth will make the result of the proposed compensation method deteriorate. Here we give the threshold of $\lambda \nu$, which is obtained from our numerical simulation. That is, we can compensate the energy loss when $\lambda \nu$ is less than 0.1. That means: (1) for using a $532 \mathrm{~nm}$ laser source, the minimum pitch of the hologram could reach $2.66 \mu \mathrm{m}$ and (2) for using current $8 \mu \mathrm{m}$ pitch $\mathrm{SLM}$, a $1600 \mathrm{~nm}$ infrared laser source could be used for $3 \mathrm{D}$ scene reconstruction with precise energy compensation.

Finally, it is worth noting that with the proposed method, the reconstruction images have speckles since, in the numerical computation, we bring a frequency band limitation to the original spectrum, which should be infinite over the entire spectral domain [19]. To mitigate the speckle, various approaches could be used [20-22].

\section{Conclusion}

An analytical brightness compensation method is proposed for the traditional polygon-based method. The surface function that has the flat PSD is used. The 3D spatial rotational transformation between the local and the global coordinates of the tilted polygon is simplified to two independent rotations about the $x, y$ coordinate axes, by which the original and the new frequencies have finite and solvable relationship. The new frequencies are further approximated by discarding the higher-order infinitesimal term to obtain the area of the effective domain. The brightness of each tilted polygon is preserved by multiplying the rotated spectrum by the compensation factor, which is the square root of the ratio between the area of the effective domain and that of the original domain. The optical experiments were carried to prove the effectiveness of the proposed approach. The reconstruction results showed the compensation method could correctly compensate the brightness difference to the $3 \mathrm{D}$ scene, which ensures the further 
shading process. The advantage of the proposed method is that it could compensate the brightness analytically excluding the influence of the fabrication process. The limitation of the proposed method is that it cannot precisely compensate the energy loss for the system which has excessive long reconstruction wavelength or high spectral bandwidth that exceeds the provided threshold. Another issue is the proposed method brings speckles in the reconstruction. In the following research, we will take the higher-order term into account and precisely compensate the energy loss when the system breaks the proposed threshold. We will also improve the compensation algorithm by considering the random signal with the different PSD, which could condense the spectrum into a finite frequency band, by which the speckle in the reconstruction will be reduced. We believe the proposed method is beneficial to deepening the investigation of the influence of the fabrication process, and it is useful for the realistic reconstruction in the holographic 3D display.

This work is supported by the National Basic Research Program of China (973 Program grants 2013CB328801 and 2013CB328806), the National Natural Science Foundation of China (61235002), and the China Scholarship Council.

\section{References}

1. C. Slinger, C. Cameron, and M. Stanley, "Computer-generated holography as a generic display technology," Computer 38, 4653 (2005).

2. M. Lucente, "Interactive computation of holograms using a look-up table," J. Electron. Imaging 2, 28-34 (1993).

3. Y. Pan, X. Xu, S. Solanki, X. Liang, R. Tanjung, C. Tan, and T.-C. Chong, "Fast CGH computation using S-LUT on GPU," Opt. Express 17, 18543-18555 (2009).

4. J. Weng, T. Shimobaba, N. Okada, H. Nakayama, M. Oikawa, N. Masuda, and T. Ito, "Generation of real-time large computer generated hologram using wavefront recording method," Opt. Express 20, 4018-4023 (2012).

5. K. Matsushima, H. Schimmel, and F. Wyrowski, "Fast calculation method for optical diffraction on tilted planes by use of the angular spectrum of plane waves," J. Opt. Soc. Am. A 20, 1755-1762 (2003).
6. K. Matsushima, "Computer-generated holograms for threedimensional surface objects with shade and texture," Appl. Opt. 44, 4607-4614 (2005).

7. K. Matsushima, "Formulation of the rotational transformation of wave fields and their application to digital holography," Appl. Opt. 47, D110-D116 (2008).

8. K. Matsushima and S. Nakahara, "Extremely high-definition full-parallax computer-generated hologram created by the polygon-based method," Appl. Opt. 48, H54-H63 (2009).

9. H. Nishi, K. Matsushima, and S. Nakahara, "Rendering of specular surfaces in polygon-based computer-generated holograms," Appl. Opt. 50, H245-H252 (2011).

10. K. Matsushima, H. Nishi, and S. Nakahara, "Simple wavefield rendering for photorealistic reconstruction in polygonbased high-definition computer holography," J. Electron. Imaging 21, 023002 (2012).

11. L. Ahrenberg, P. Benzie, M. Magnor, and J. Watson, "Computer generated holograms from three dimensional meshes using an analytic light transport model," Appl. Opt. 47, 1567-1574 (2008).

12. H. Kim, J. Hahn, and B. Lee, "Mathematical modeling of triangle-mesh-modeled three-dimensional surface objects for digital holography," Appl. Opt. 47, D117-D127 (2008).

13. H. Sakata and Y. Sakamoto, "Fast computation method for a Fresnel hologram using three-dimensional affine transformations in real space," Appl. Opt. 48, H212-H221 (2009).

14. K. Hosoyachi, K. Yamaguchi, T. Ichikawa, and Y. Sakamoto, "Precalculation method using spherical basic object light for computer-generated hologram," Appl. Opt. 52, A33-A44 (2013).

15. Y. Pan, Y. Wang, J. Liu, X. Li, and J. Jia, "Fast polygon-based method for calculating computer-generated holograms in three-dimensional display,” Appl. Opt. 52, A290-A299 (2013).

16. J. W. Goodman, Introduction to Fourier Optics (McGraw-Hill, 1996).

17. J. D. Foley, A. van Dam, S. K. Feiner, and J. F. Hughes, Computer Graphics: Principles and Practice, Second Edition in $C$ (Addison-Wesley, 1996).

18. R. Hartley and A. Zisserman, Multiple View Geometry in Computer Vision, 2nd ed. (Cambridge University, 2003).

19. R. Bräuer, F. Wyrowski, and O. Bryngdahl, "Diffusers in digital holography," J. Opt. Soc. Am. A 8, 572-578 (1991).

20. F. Wyrowski and O. Bryngdahl, "Speckle-free reconstruction in digital holography," J. Opt. Soc. Am. A 6, 1171-1174 (1989).

21. J. Amako, H. Miura, and T. Sonehara, "Speckle-noise reduction on kinoform reconstruction using a phase-only spatial light modulator," Appl. Opt. 34, 3165-3171 (1995).

22. J. I. Trisnadi, "Hadamard speckle contrast reduction," Opt. Lett. 29, 11-13 (2004). 